\title{
MODEL BASED DIAGNOSIS OF THE AIR PATH OF AN AUTOMOTIVE DIESEL ENGINE
}

\author{
Mattias Nyberg ${ }^{1}$, Thomas Stutte ${ }^{2}$, and Volker Wilhelmi ${ }^{3}$
}

\author{
DaimlerChrysler AG, Research and Technology (FT2/EA) \\ HPC:T721, D-70546 Stuttgart, Germany \\ Email:matny@isy.liu.se ${ }^{1}$, thomas.stutte@daimlerchrysler.com ${ }^{2}$, \\ volker.wilhelmi@daimlerchrysler.com ${ }^{3}$
}

\begin{abstract}
A model based diagnosis system for the air-path of a turbo-charged diesel engine with EGR is constructed. The faults considered were air-mass flow sensor fault, intake-manifold pressure sensor fault, air-leakage, and the EGR-valve stuck in closed position. A non-linear engine model, with four states, is constructed. The diagnosis system is then constructed in the framework of structured hypothesis tests, and by using adaptive observers. The diagnosis system is successfully evaluated in a real car driving on the road.
\end{abstract}

Keywords: Diagnosis, Fault Detection, On-Board Diagnostics, Diesel Engine, Modeling, Hypothesis Testing, Adaptive Observers

\section{INTRODUCTION}

Since the year 1996, cars sold in California are required to have an on-board diagnosis (OBD) system. These requirements, called OBDII (On Board Diagnostics), were introduced by CARB (California Air Resource Board). The same type of requirements have since then also been introduced in the other parts of USA. Further, from year 2000, all cars sold in EU must be equipped with an OBD system. Automotive diesel engines are not sold so much in the USA, but they are very popular in EU. Therefore, the importance of OBD systems for diesel engines has increased significantly since year 2000 .

One important part of the diagnosis requirements for diesel engines is the components in the air path. Possible faults includes sensor faults, actuator faults, and leakages. These types of faults typically lead to degraded emission control, and also possible damage to engine components.

In this paper we consider diagnosis of faults in the air-path of a turbo-charged diesel engine. The engine is equipped with EGR (Exhaust Gas Recycling) and a VNT (Variable Nozzle Turbine). The faults considered are air-mass flow sensor fault, intakemanifold pressure sensor fault, air-leakage between the air-mass flow sensor and the cylinders, and the EGR-valve stuck in closed position.

The solutions presented here are based on previous works presented in (Nyberg and Perkovic, 1998; Nyberg, 1999a). However, the engine investigated in the previous works did not include EGR and VNT. These both components makes the diagnosis problem much more difficult since the air flow through the EGRvalve, and also the exhaust side of the engine have to be taken into account. Thus the main challenge in this paper is to construct a diagnosis system for an engine which includes EGR and VNT.
In Sections 2 and 3, the engine and a model of the engine are presented. Then, in Section 4, models of the different faults are presented. The diagnosis system is constructed within the framework of structured hypothesis tests (Nyberg, 2000) and the basic principles for this are given in Section 5. Then the actual design of the diagnosis system is covered in Section 6. Finally, Section 7 describes experiments on a real vehicle.

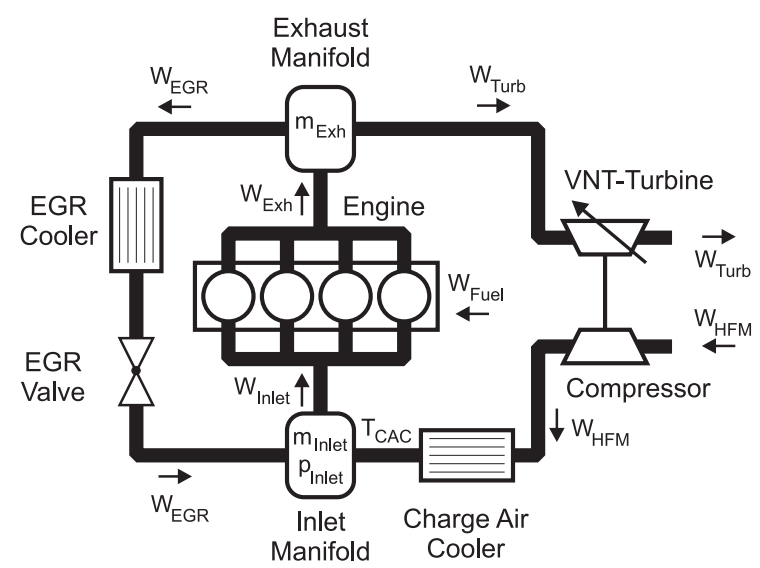

Fig. 1. The Mercedes-Benz OM611 diesel engine.

\section{ENGINE DESCRIPTION}

The diesel engine is a Mercedes-Benz OM611, has 2.2 liter displacement, and direct ignition. Detailed information about the engine can be found in (Klingmann. et al., 1999). A principle illustration is shown in Figure 1. The air entering the engine is measured by an air-mass flow-meter (HFM). It then passes the compressor and the CAC (Charge Air Cooler), enters the intake manifold, and flows into the cylinders. On the exhaust side, the exhausts are partly driving the turbine, and are partly recycled via the EGR (Exhaust Gas Recycling) path. 
The production version of the engine is equipped with sensors measuring in-flowing air $W_{H F M}$, the temperature after CAC $T_{C A C}$, and inlet manifold pressure $P_{\text {Inlet }}$. The inputs to the engine are $W_{\text {Fuel }}$, the turbine vane position $X_{V N T}$, and the EGR-valve position $A_{E G R}$. In the model, also $N_{E n g}$ is considered to be an input.

\begin{tabular}{|c|c|c|}
\hline variable & unit & explanation \\
\hline$p_{\text {Inlet }}$ & $P a$ & pressure in intake manifold \\
\hline$V_{\text {Inlet }}$ & $m^{3}$ & volume of intake manifold \\
\hline$R_{A i r}$ & $J /(k g \cdot K)$ & gas constant of air \\
\hline$c_{p, A i r}$ & $J /(k g \cdot K)$ & $\begin{array}{l}\text { specific heat at const. pres. } \\
\text { of air }\end{array}$ \\
\hline$c_{v}$, Air & $J /(k g \cdot K)$ & $\begin{array}{l}\text { specific heat at const. vol. } \\
\text { of air }\end{array}$ \\
\hline$R_{E x h}$ & $J /(k g \cdot K)$ & gas constant of exhaust gas \\
\hline$c_{p, E x h}$ & $J /(k g \cdot K)$ & $\begin{array}{l}\text { specific heat at const. pres. } \\
\text { of exhaust gas }\end{array}$ \\
\hline$c_{v, E x h}$ & $J /(k g \cdot K)$ & $\begin{array}{l}\text { specific heat at const. vol. } \\
\text { of exhaust gas }\end{array}$ \\
\hline$R_{\text {Inlet }}$ & $J /(k g \cdot K)$ & $\begin{array}{l}\text { gas constant of gas in intake } \\
\text { manifold }\end{array}$ \\
\hline$c_{p, \text { Inlet }}$ & $J /(k g \cdot K)$ & $\begin{array}{l}\text { specific heat at const. pres. } \\
\text { of gas in intake manifold }\end{array}$ \\
\hline$c_{v, \text { Inlet }}$ & $J /(k g \cdot K)$ & $\begin{array}{l}\text { specific heat at const. vol. } \\
\text { of gas in intake manifold }\end{array}$ \\
\hline$\kappa$ & $c_{p} / c_{v}$ & ratio of specific heats \\
\hline$W_{H F M}$ & $\mathrm{~kg} / \mathrm{s}$ & $\begin{array}{l}\text { air mass-flow past the air } \\
\text { mass-flow sensor }\end{array}$ \\
\hline$T_{C A C}$ & $K$ & $\begin{array}{l}\text { temperature of the air after } \\
\text { the charge-air cooler }\end{array}$ \\
\hline$W_{E G R}$ & $\mathrm{~kg} / \mathrm{s}$ & $\begin{array}{l}\text { EGR mass-flow into intake } \\
\text { manifold }\end{array}$ \\
\hline$T_{E G R}$ & $K$ & $\begin{array}{l}\text { temperature of EGR gas- } \\
\text { flow into the intake manifold }\end{array}$ \\
\hline$W_{\text {Inlet }}$ & $\mathrm{kg} / \mathrm{s}$ & $\begin{array}{l}\text { mass-flow into engine inlet } \\
\text {-ports }\end{array}$ \\
\hline$T_{\text {Inlet }}$ & $K$ & $\begin{array}{l}\text { temperature in the intake } \\
\text { manifold }\end{array}$ \\
\hline$m_{\text {Air }}$ & $k g$ & mass of air in intake manifold \\
\hline$m_{E G R}$ & $k g$ & $\begin{array}{l}\text { mass of EGR-gas in intake } \\
\text { manifold }\end{array}$ \\
\hline$W_{E x h}$ & $\mathrm{~kg} / \mathrm{s}$ & $\begin{array}{l}\text { exhaust mass-flow into the } \\
\text { exhaust manifold }\end{array}$ \\
\hline$m_{E x h}$ & $k g$ & $\begin{array}{l}\text { mass of exhaust gas in } \\
\text { exhaust manifold }\end{array}$ \\
\hline$p_{E x h}$ & $P a$ & pressure in exhaust manifold \\
\hline$T_{E x h}$ & $K$ & $\begin{array}{l}\text { temperature in exhaust } \\
\text { manifold }\end{array}$ \\
\hline$A_{E G R}$ & $m^{2}$ & effective area of EGR valve \\
\hline$V_{E n g}$ & $m^{3}$ & engine displacement \\
\hline$N_{E n g}$ & $\min ^{-1}$ & engine speed \\
\hline$W_{\text {Fuel }}$ & $\mathrm{kg} / \mathrm{s}$ & mass-flow of injected fuel \\
\hline$Q_{L H V}$ & $\mathrm{~J} / \mathrm{kg}$ & Lower Heating Value \\
\hline$V_{E x h}$ & $m^{3}$ & volume of exhaust manifold \\
\hline$p_{A t m}$ & $P a$ & atmospheric pressure \\
\hline$W_{T u r b}$ & $\mathrm{~kg} / \mathrm{s}$ & $\begin{array}{l}\text { exhaust mass-flow past } \\
\text { the turbine }\end{array}$ \\
\hline$X_{V N T}$ & $\%$ & position of VNT vanes \\
\hline
\end{tabular}

\section{MODELING}

The model used in the diagnosis algorithm is based on principles described in (Heywood, 1992; Guzzella and Amstutz, 1998; Nyberg and Perkovic, 1998; Truscott et al., 2000). For diagnosing the faults of interest, it is not necessary to include the compressor and the $\mathrm{CAC}$ in the model. The reason for this is that no faults in the compressor or the CAC are considered, and also, the mass-flow and the temperature after the CAC are already known variables because they are measured by the production sensors.
Table 1 lists the variables used in the model. The model, for the fault-free case, is as follows:

$$
\begin{aligned}
\dot{p}_{\text {Inlet }}= & \frac{1}{V_{\text {Inlet }}}\left(\frac{R_{\text {Air }} c_{p, \text { Air }}}{c_{v, \text { Air }}} W_{H F M} T_{C A C}+\right. \\
& +\frac{R_{E x h} c_{p, E x h}}{c_{v, E x h}} W_{E G R} T_{E G R} \\
& \left.-\frac{R_{\text {Inlet }} c_{p, \text { Inlet }}}{c_{v, \text { Inlet }}} W_{\text {Inlet }} T_{\text {Inlet }}\right) \\
\dot{m}_{\text {Air }}= & W_{H F M}-\frac{m_{\text {Air }}}{m_{\text {Air }}+m_{E G R}} W_{\text {Inlet }} \\
\dot{m}_{E G R}= & W_{E G R}-\frac{m_{E G R}}{m_{\text {Air }}+m_{E G R}} W_{\text {Inlet }} \\
\dot{m}_{E x h}= & W_{E x h}-W_{\text {Turb }}-W_{E G R}
\end{aligned}
$$

where

$$
\begin{aligned}
& W_{E G R}=\frac{A_{E G R} p_{E x h}}{\sqrt{R_{E x h} T_{E x h}}} \Psi_{\kappa_{E x h}}\left(\frac{p_{\text {Inlet }}}{p_{E x h}}\right) \\
& \Psi_{\kappa}\left(\frac{p_{1}}{p_{0}}\right)=\left\{\begin{array}{l}
\sqrt{\frac{2 \kappa}{\kappa-1}\left\{\left(\frac{p_{1}}{p_{0}}\right)^{\frac{2}{\kappa}}-\left(\frac{p_{1}}{p_{0}}\right)^{\frac{\kappa+1}{\kappa}}\right\}_{\kappa}} \\
\text { if }\left(\frac{p_{1}}{p_{0}}\right) \geq\left(\frac{2}{\kappa+1}\right)^{\frac{\kappa}{\kappa-1}} \\
\sqrt{\kappa\left(\frac{2}{\kappa+1}\right)^{\frac{\kappa+1}{\kappa-1}}} \quad \text { otherwise }
\end{array}\right.
\end{aligned}
$$

$$
W_{\text {Inlet }}=f_{\text {vol }}\left(N_{\text {Eng }}, \frac{p_{\text {Inlet }}}{T_{\text {Inlet }} R_{\text {Inlet }}}\right) \frac{N_{\text {Eng }} p_{\text {Inlet }}}{T_{\text {Inlet }} R_{\text {Inlet }}} \frac{V_{\text {Eng }}}{120}
$$$$
T_{\text {Inlet }}=\frac{p_{\text {Inlet }} V_{\text {Inlet }}}{\left(m_{\text {Air }}+m_{E G R}\right) R_{\text {Inlet }}}
$$$$
T_{E G R}=831 K
$$$$
W_{\text {Exh }}=W_{\text {Inlet }}+W_{\text {Fuel }}
$$$$
T_{\text {Exh }}=T_{\text {Inlet }}+\frac{Q_{L H V} h\left(W_{\text {Fuel }}, N_{\text {Eng }}\right)}{c_{p, E x h}\left(W_{\text {Inlet }}+W_{\text {Fuel }}\right)}
$$$$
p_{E x h}=\frac{m_{E x h} R_{E x h} T_{E x h}}{V_{E x h}}
$$$$
R_{\text {Inlet }}=\frac{R_{\text {Air }} m_{\text {Air }}+R_{E x h} m_{E G R}}{m_{\text {Air }}+m_{E G R}}
$$$$
c_{v, \text { Inlet }}=\frac{c_{v, \text { Air }} m_{A i r}+c_{v, E x h} m_{E G R}}{m_{A i r}+m_{E G R}}
$$$$
c_{p, \text { Inlet }}=c_{v, \text { Inlet }}+R_{\text {Inlet }}
$$$$
W_{\text {Turb }}=\frac{p_{E x h}}{\sqrt{T_{E x h}}} g\left(\frac{p_{E x h}}{p_{A t m}}, X_{V N T}\right)
$$

As seen, the temperature $T_{E G R}$ is assumed to be constant. The model contains three static functions: $f_{v o l}, h$, and $g$. They are represented as interpolation in lookup tables (maps). The parameters and lookup tables in the model, were obtained partly from manufacturer data, and partly from steady state and dynamic measurements.

The presented model is assumed to be valid in the fault-free case. When there is a fault present, other models are valid and these will be described later in Section 4.

\subsection{Model Validation}

The model described above is simulated and a comparison with real measurement data can be seen in 


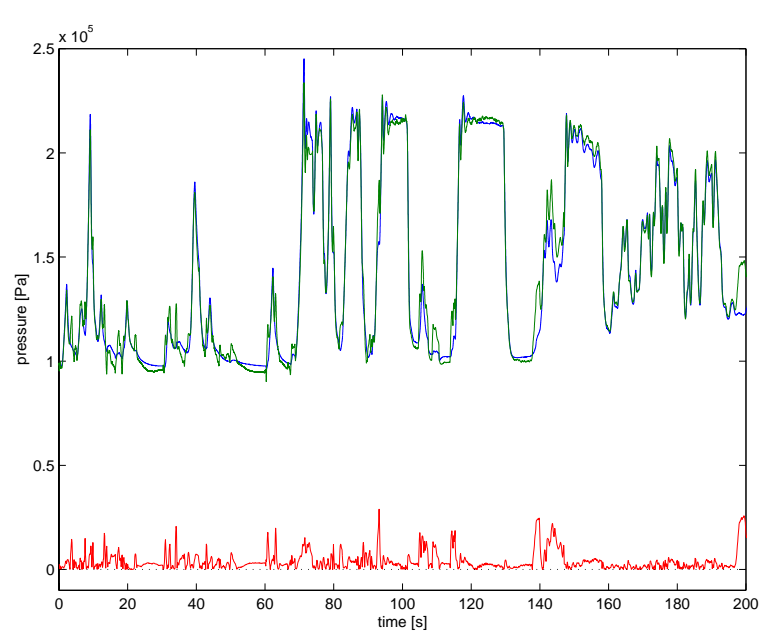

Fig. 2. Simulated and measured inlet pressure $p_{\text {Inlet }}$ (upper lines). The absolute value of the difference (lower line).

Figure 2. The figure shows the simulated and measured inlet pressure $p_{\text {Inlet }}$. The agreement is quite good but some model errors can be seen for highly dynamic parts. Also longer measurements were compared and on average, an RMS error of $3 \%$ was obtained.

\section{MODELING OF FAULTS}

The diagnosis algorithm described later in this paper uses the framework of structured hypothesis tests proposed in (Nyberg, $1999 a$; Nyberg, 1999b; Nyberg, 2000). In this framework, it is possible to utilize detailed models for the faults. Below, the different models, for the faults considered in this paper, are described. However, first we introduce the concept of fault modes.

\subsection{Fault Modes}

When using structured hypothesis tests, the different faults are classified into different fault modes. We consider the following fault modes:

\begin{tabular}{ll}
\hline Abbreviation & Explanation \\
\hline$N F$ & no fault \\
$H F M$ & air-mass flow sensor fault \\
$I P S$ & intake-manifold pressure sensor fault \\
$M L$ & air-leakage between the air-mass flow \\
& sensor and the cylinders \\
$E G R$ & EGR-valve is stuck in closed position \\
\hline
\end{tabular}

Note that one of the fault modes is the no-fault case. This list of fault modes was chosen based on how often the faults occur, how difficult they are to diagnose with the current non-model based algorithms, and how big effects they have on the system. Note however, that no specific investigations have been made regarding how these different faults influence the emissions. Each fault mode is associated with a model of the engine. Next, each of the models for all the fault modes are described.

4.1.1. No Fault (NF) The model for the fault free engine was described in Section 3. To completely describe the $N F$ fault-mode, we also add equations describing that also the sensors and actuators are fault free:

$$
\begin{aligned}
W_{H F M, s} & =W_{H F M} \\
T_{\text {Inlet }, s} & =T_{\text {Inlet }} \\
p_{\text {Inlet }, s} & =p_{\text {Inlet }} \\
N_{\text {Eng }, s} & =N_{\text {Eng }} \\
p_{\text {Atm }, s} & =p_{\text {Atm }} \\
A_{E G R} & =A_{E G R, r} \\
W_{F u e l} & =W_{F u e l, r} \\
X_{V N T} & =X_{V N T, r}
\end{aligned}
$$

Here, index $s$ indicates sensored value and index $r$ indicates reference value, set by the controller. For example, equation (17a) says that the measured value $W_{H F M, s}$ is equal to the physical variable $W_{H F M}$.

4.1.2. Air Mass-Flow Sensor Fault (HFM) The model for the fault mode $H F M$ is obtained by taking the model for the $N F$ fault-mode but replacing equation (17a) with

$$
W_{H F M, s}=g W_{H F M}
$$

where $g$ is an unknown constant, and $g \neq 1$.

\subsection{Inlet-Pressure Sensor Fault (IPS)}

The model for the fault mode IPS is obtained by taking the model for the $N F$ fault-mode but replacing equation $(17 \mathrm{c})$ with

$$
p_{\text {Inlet }, s}=k p_{\text {Inlet }}
$$

where $k$ is an unknown constant, and $k \neq 1$.

\subsection{Manifold Leakage (ML)}

The leakage size is assumed constant and the flow through the leakage is modeled as a flow through a restriction. This type of model has been validated in (Nyberg and Perkovic, 1998) with good results.

Equations (1), (2), and (3) in the fault-free model is replaced by

$$
\begin{aligned}
\dot{p}_{\text {Inlet }}= & \frac{1}{V_{\text {Inlet }}}\left(\frac{R_{\text {Air }} c_{p, \text { Air }}}{c_{v, \text { Air }}} W_{H F M} T_{C A C}+\right. \\
& +\frac{R_{E x h} c_{p, E x h}}{c_{v, E x h}} W_{E G R} T_{E G R} \\
& \left.-\frac{R_{\text {Inlet }} c_{p, \text { Inlet }}}{c_{v, \text { Inlet }}}\left(W_{\text {Inlet }}+W_{\text {Leak }}\right) T_{\text {Inlet }}\right) \\
\dot{m}_{\text {Air }}= & W_{H F M}-\frac{m_{\text {Air }}}{m_{\text {Air }}+m_{E G R}}\left(W_{\text {Inlet }}+W_{\text {Leak }}\right) \\
\dot{m}_{E G R}= & W_{E G R}-\frac{m_{E G R}}{m_{\text {Air }}+m_{E G R}}\left(W_{\text {Inlet }}+W_{\text {Leak }}\right)
\end{aligned}
$$

where

$$
W_{\text {Leak }}=\frac{A_{\text {Leak }} p_{\text {Inlet }}}{\sqrt{R_{\text {Inlet }} T_{\text {Inlet }}}} \Psi_{\kappa_{\text {Air }}}\left(\frac{p_{\text {Atm }}}{p_{\text {Inlet }}}\right)
$$

and the function $\Psi_{\kappa}(\cdot)$ was defined in (6). All sensors and actuators are assumed fault free, and thus equations (17) are assumed to hold.

\subsection{EGR-Valve Stuck Closed (EGR)}

When the EGR valve is stuck in closed position, no gas flow is able to pass the EGR valve. Thus, the model for the fault mode $E G R$ is obtained by taking the model for the $N F$ fault-mode but replacing equation (17f) with

$$
A_{E G R}=0
$$




\subsection{A One-Model-View}

Above, we have defined 5 different models for each of the 5 different fault modes. It is also possible to view this as one model with a free vector valued parameter. This is advantageous when later, in Section 5, the diagnosis problem is described from the view of hypothesis testing.

First, introduce the variable $q$ and let equation (18) be written

$$
A_{E G R}=q A_{E G R, r} \quad q=0
$$

With the vector valued parameter $\theta=\left[\begin{array}{ll}g k A_{\text {Leak }} q\end{array}\right]$, each of the 5 different models can be obtained from one model $\mathcal{M}(\theta)$ by letting $\theta$ be constrained to a certain set $\Theta_{\gamma}$, where $\gamma$ denotes the corresponding fault mode. For example, the $N F$ fault-mode is obtained when $\theta=\left[\begin{array}{llll}1 & 1 & 0 & 1\end{array}\right]$. All the sets $\Theta_{\gamma}$ corresponding to each of the fault modes are

$$
\begin{aligned}
& \Theta_{N F}=\left\{\left[\begin{array}{llll}
1 & 1 & 0 & 1
\end{array}\right]\right\} \\
& \Theta_{H F M}=\left\{\left[\begin{array}{llll}
g & 1 & 0 & 1
\end{array}\right] \mid g \neq 1\right\} \\
& \Theta_{I P S}=\left\{\left[\begin{array}{llll}
1 & k & 0 & 1
\end{array}\right] \mid k \neq 1\right\}
\end{aligned}
$$

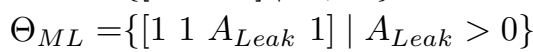

$$
\begin{aligned}
& \Theta_{E G R}=\left\{\left[\begin{array}{llll}
1 & 1 & 0 & 0
\end{array}\right]\right\}
\end{aligned}
$$

\section{ISOLATION USING STRUCTURED HYPOTHESIS TESTS}

With the fault modes defined above, the diagnosis problem can be stated as follows:

Given measured data, which different models, defined by the different fault modes, can explain the measured data?

This means, that the task of diagnosis is to investigate which of the different models that match the measured data. Because of model errors and measurement noise, a formalized decision procedure for this is naturally based on hypothesis testing. To construct the diagnosis system by combining a set of hypothesis tests is the basic idea of structured hypothesis tests. The hypothesis tests used are "binary", i.e. the task is to test one null hypothesis against one alternative hypothesis, see e.g. (Lehmann, 1986; Casella and Berger, 1990).

Let $F_{p}$ denote the present fault mode. To describe the $k$ :th hypothesis test, introduce the set $M_{k}$. The null hypothesis and the alternative hypothesis can then be written

$H_{k}^{0}: F_{p} \in M_{k} \quad$ "some fault mode in $M_{k}$ can explain meas. data" $H_{k}^{1}: F_{p} \in M_{k}^{C}$ "no fault mode in $M_{k}$ can explain meas. data"

The convention used here and also commonly used in hypothesis testing literature, is that when $H_{k}^{0}$ is rejected, we assume that $H_{k}^{1}$ is true. This implies that the present fault mode can not belong to $M_{k}$, i.e. it must belong to $M_{k}^{C}$. Further, when $H_{k}^{0}$ is not rejected, we will not assume anything ${ }^{1}$. In this way, each hypothesis test contributes with a piece of information regarding which fault modes that can be matched to the data or not. This piece of information, i.e. the decision reached by each hypothesis test, is called a sub-diagnosis statement.

1 Sometimes it is possible to assume something also when $H_{k}^{0}$ is not rejected, see NybergPhD.
How a set of different hypothesis tests are used to diagnose and isolate faults is illustrated by the following example.

Example 1. Assume that the diagnosis system contains the following set of three hypothesis tests:

$H_{1}^{0}: F_{p} \in M_{1}=\left\{N F, F_{1}\right\} \quad H_{1}^{1}: F_{p} \in M_{1}^{C}=\left\{F_{2}, F_{3}\right\}$

$H_{2}^{0}: F_{p} \in M_{2}=\left\{N F, F_{2}\right\} \quad H_{2}^{1}: F_{p} \in M_{2}^{C}=\left\{F_{1}, F_{3}\right\}$

$H_{3}^{0}: F_{p} \in M_{3}=\left\{N F, F_{3}\right\} \quad H_{3}^{1}: F_{p} \in M_{3}^{C}=\left\{F_{1}, F_{2}\right\}$

Then if only $H_{1}^{0}$ is rejected, we can draw the conclusion that $F_{p} \in M_{1}^{C}=\left\{F_{2}, F_{3}\right\}$, i.e. the present fault mode is either $F_{2}$ or $F_{3}$. If both $H_{1}^{0}$ and $H_{2}^{0}$ are rejected, we can draw the conclusion that $F_{p} \in M_{1}^{C} \cap$ $M_{2}^{C}=\left\{F_{2}, F_{3}\right\} \cap\left\{F_{1}, F_{3}\right\}=\left\{F_{3}\right\}$, i.e. the present fault mode is $F_{3}$.

The decision reached by the whole diagnosis system is called the diagnosis statement. From the example above, it is clear that to find the diagnosis statement from the sub-diagnoses statements, a simple intersection operation can be used. To develop the actual hypothesis tests, we first need to decide the set of hypotheses to test. One solution is to use one hypothesis test for each fault mode.

\subsection{Hypothesis Tests}

Each hypothesis test can also be described by using the sets $\Theta_{\gamma}$, defined in Section (4.5). This is done via sets $\Theta_{k}^{0}$ which are defined as

$$
\Theta_{k}^{0}=\bigcup_{\gamma \in M_{k}} \Theta_{\gamma}
$$

The hypotheses can now be expressed as

$\begin{array}{ll}H_{k}^{0}: \theta \in \Theta_{k}^{0} & \text { "some value of } \theta \in \Theta_{k}^{0} \text { can explain meas. data" } \\ H_{k}^{1}: \theta \notin \Theta_{k}^{0} & \text { "no value of } \theta \in \Theta_{k}^{0} \text { can explain meas. data" }\end{array}$

For each hypothesis test, we need to find a test quantity and a threshold. The test quantity is a function $T_{k}(\mathbf{x})$ from the sample data $\mathbf{x}$, to a scalar value which is to be thresholded by the threshold $J_{k}$. The sample data $\mathbf{x}$ can be all measured data up to present time or a subset of this data. One choice is to use a fixed size time window. If $T_{k}(\mathbf{x}) \geq J_{k}$, then $H_{k}^{0}$ is rejected and otherwise not rejected.

The test quantity $T_{k}(\mathbf{x})$ is in many texts instead called a test statistic. However, the name test statistic indicates that $T_{k}(\mathbf{x})$ is a random variable which in general may not be a desired view. In many applications, a deterministic view is taken and $T_{k}(\mathbf{x})$ is seen just as a function of the data and not as a random variable.

According to what has been said above, we need to design each test quantity $T_{k}(\mathbf{x})$ such that it is low or at least below the threshold if the data $\mathbf{x}$ matches the hypothesis $H_{k}^{0}$, i.e. a fault mode in $M_{k}$ can explain the data. Using traditional fault-diagnosis terminology, the fault modes in $M_{k}$ are said to be decoupled. Also if the data come from a fault mode not in $M_{k}, T_{k}(\mathbf{x})$ should be large or at least above the threshold.

To be able to make the assumption that $H_{k}^{1}$ is true when $H_{k}^{0}$ is rejected, we need to design the hypothesis 
test such that the significance level is small. This implies that the threshold $J_{k}$ must be set relatively high. This in turn means that the value of the power function $P$ (reject $H_{k}^{0} \mid \theta$ ) does not necessarily become large for all values $\theta \notin \Theta_{k}^{0}$. For instance, if the present fault mode is $F_{i}$ and $F_{i} \in M_{k}^{C}$, then for some $\theta \in \Theta_{F_{i}}$, the probability to reject $H_{k}^{0}$ may be very small. This is the reason why we don't assume anything when $H_{k}^{0}$ is not rejected.

It turns out that some fault modes are related to other system fault modes such that for some values of $\theta$ they are impossible to separate. These relations between fault modes have implications on how the sets $M_{k}$ (i.e. the null hypotheses) can be chosen. For example for most fault modes, the limit when the fault size goes to zero is equal to the fault mode "no fault". This means that when fault mode $N F$, i.e. no fault, is present, most null hypothesis can not be rejected. The implication is that almost all sets $M_{k}$ must include $N F$. For more information on this relation and how it affects the choice of null hypotheses, see (Nyberg, 1999b).

\section{DESIGN OF THE DIAGNOSIS SYSTEM}

In the previous section, some general principles for constructing the diagnosis system were discussed. In this section we go into the actual design of the diagnosis system for the diesel engine.

\subsection{Choice of Null Hypotheses}

We chose to use one hypothesis test for each of the fault modes. However, because of the submode relation mentioned in Section 5.1, the NF fault mode must be included in almost all null hypotheses. This means that the complete list of null hypotheses is defined by the following $M_{k}$-sets:

$$
\begin{aligned}
& M_{1}=\{N F\} \\
& M_{2}=\{N F, H F M\} \\
& M_{3}=\{N F, I P S\} \\
& M_{4}=\{N F, M L\} \\
& M_{5}=\{E G R\}
\end{aligned}
$$

Also without the first hypothesis test, with $M_{1}=$ $\{N F\}$, it is possible to perform isolation. However, to save computational time, it can be desirable to first execute the $N F$-test. Then only if this null hypothesis, i.e. $H_{1}^{0}: F_{p}=N F$, is rejected, the other hypothesis tests are executed.

\subsection{Construction of Test Quantities}

The test quantities are calculated as

$$
T_{k}=\frac{1}{t_{1}-t_{0}} \int_{t_{0}}^{t_{1}} r_{k}^{2}(t) d t
$$

where $r_{k}(t)$ is the residual between measured and the estimated pressure $p_{\text {Inlet }}$. The window length, i.e. the interval $\left[t_{o}, t_{1}\right]$, is chosen to be 1 minute.

For the fault modes $N F$ and EGR, the corresponding models contain no unknown parameters. This means that the residuals $r_{k}(t)$ can simply be calculated as

$$
r_{k}(t)=p_{\text {Inlet }, s}(t)-\hat{p}_{\text {Inlet }, k}(t)
$$

where $\hat{p}_{\text {Inlet }, k}(t)$ is calculated by simulating the model.

For the fault modes $H F M, I P S$, and $M L$, each of the corresponding models contains one unknown parameter. Let this parameter be denoted $\theta_{k}$. Then the residual $r_{k}$ is dependent on $\theta_{k}$ and we can write

$$
r_{k}\left(t \mid \theta_{k}\right)=p_{\text {Inlet }, s}(t)-\hat{p}_{\text {Inlet }, k}\left(t \mid \theta_{k}\right)
$$

That is, when calculating the estimated pressure $\hat{p}_{\text {Inlet }, k}\left(t \mid \theta_{k}\right)$, it is first necessary to assume a value of $\theta_{k}$. The solution in this work is to use an adaptive observer get the estimate $\hat{\theta}_{k}$. Then $\hat{p}_{\text {Inlet }, k}\left(t \mid \theta_{k}\right)$ can be calculated by simulating the model with $\theta_{k}=\hat{\theta}_{k}$. In other words, first the collected data (i.e. the sample data $\mathbf{x}$ ) is used to estimate $\hat{\theta}_{k}$, and then, the same data is used to calculate the value of the test quantity $T_{k}(\mathbf{x})$

\subsection{Adaptive Observers}

Define $\mathbf{z}=\left[p_{\text {Inlet }}, m_{\text {Air }}, m_{E G R}, m_{E x h}\right]$ and $\mathbf{u}=$ $\left[W_{\text {Fuel }}, N_{E n g}, X_{V N T}, A_{E G R}\right]$. Also, let $\theta_{k}$ denote the part of the $\theta$-vector that is to be estimated. The system dynamics, including a state for $\theta_{k}$, can then be written in state-space form as

$$
\begin{aligned}
\dot{\mathbf{z}} & =\mathbf{f}\left(\mathbf{z}, \mathbf{u}, \theta_{k}\right) \\
\dot{\theta}_{k} & =0
\end{aligned}
$$

The system is assumed stable, and also it is assumed that we can estimate the initial values of the states reasonable well. Therefore no feedback is used for the states $\mathbf{z}$. The adaptive observers are then constructed as

$$
\begin{aligned}
\dot{\hat{\mathbf{z}}} & =\mathbf{f}_{k}\left(\hat{\mathbf{z}}, \mathbf{u}, \hat{\theta}_{k}\right) \\
\dot{\hat{\theta_{k}}} & =G_{k}\left(p_{\text {Inlet }, s}-\hat{p}_{\text {Inlet }}\right) \\
\hat{p}_{\text {Inlet }} & =z_{1}
\end{aligned}
$$

\subsection{Normalization of the Test Quantities}

Because there are model errors present, it is advantageous to use a normalization of the test quantities. This idea has also been used in (Nyberg, 1999a) with good results. Each test quantity is normalized as follows:

$$
T_{k}^{n o r m}=T_{k}-\mu \min _{k} T_{k}
$$

where the constant $\mu \in[0,1]$ determines how "much" normalization that should be used. The idea is that when the engine is run in an operating condition where the model has a large model error, then $\min _{k} T_{k}$ becomes quite large. This reduces the size of the normalized test quantity $T_{k}^{n o r m}$ and thus, a false alarm is avoided. As is explained in (Nyberg, 1999b), the idea is similar to the maximum likelihood ratio for stochastic systems, and adaptive thresholds, see e.g. (Höfling and Isermann, 1996).

\section{EXPERIMENTAL RESULTS}

To test the diagnosis algorithm, test sequences of one-minute measurements were used. The measurements were collected from a real car, driving on the road. Sensor faults were simulated in the computer while leakages and EGR faults were implemented in the car. The sensor-fault sizes were $g=0.9$ for the $H F M$ fault mode, and $k=1.1$ for the $I P S$ fault mode, and the leakage-sizes were $4 \mathrm{~mm}$ and $6 \mathrm{~mm}$ in diameter.

For each fault case, a total of 11 one-minute measurements were used. For each fault case and each one-minute measurement, a diagnosis statement was calculated. This means that in total, 66 cases were tested. An example is shown in Table 2, which lists all the test-quantity values and the sub-diagnosis 
statements obtained in an experiment when the $H F M$ fault-mode was present. For each row, the subdiagnosis statement is the complement of the $M_{k}$-set if the test quantity $T_{k}^{\text {norm }}$ is above the threshold $J_{k}$, and otherwise the set of all fault modes.

\begin{tabular}{llll}
$M_{k}$ & $T_{k}^{\text {norm }}$ & $J_{k}$ & sub-diagnosis statement \\
\hline$\{N F\}$ & 0.4921 & 0.4 & $\{I P S, H F M, M L, E G R\}$ \\
$\{N F, H F M\}$ & 0.098 & 0.4 & $\{N F, I P S, H F M, M L, E G R\}$ \\
$\{N F, I P S\}$ & 0.1481 & 0.4 & $\{N F, I P S, H F M, M L, E G R\}$ \\
$\{N F, M L\}$ & 0.423 & 0.4 & $\{I P S, H F M, E G R\}$ \\
$\{E G R\}$ & 0.528 & 0.4 & $\{N F, I P S, H F M, M L\}$ \\
\hline Diagnosis Statement: & $\{I P S, H F M\}$
\end{tabular}

Table 2. The hypothesis tests and the diagnosis statement for an experiment with fault mode $H F M$ present.

\begin{tabular}{ll}
\hline $\begin{array}{l}\text { present } \\
\text { fault-mode }\end{array}$ & diagnosis statements \\
\hline$N F$ & $9\{N F, I P S, H F M, M L\}$, \\
& $2\{N F, I P S, H F M, M L, E G R\}$ \\
$H F M$ & $2\{H F M\}, 8\{I P S, H F M\}, 1\{M L\}$ \\
$I P S$ & $11\{I P S\}$ \\
$M L 4 \mathrm{~mm}$ & $6\{M L\}, 3\{M L, H F M\}, 1\{M L, I P S\}$, \\
& $1\{M L, I P S, H F M\}$ \\
$M L 6 \mathrm{~mm}$ & $11\{M L\}$ \\
$E G R$ & $11\{E G R\}$ \\
\hline
\end{tabular}

Table 3. The diagnosis statements obtained in the different experiments.

A summary of the diagnoses obtained in all experiments is shown in Table 3. In the table, we can read that when the $N F$ fault mode was present, the diagnosis statement always includes $N F$ but also other fault modes. The desired result is that $N F$ should be included, and it doesn't matter if also other fault modes are included. The explanation for this is that except for $N F$, for example a very small HFMfault can also explain the measured data. In this application, which includes noise and model errors, it is not possible to obtain the diagnosis statement $N F$ because very small faults can never be distinguished from $N F$.

For the cases when other faults modes were present, a non-unique diagnosis statement was sometimes obtained. For example when the $H F M$ fault mode was present, the diagnosis statement was $\{H F M\}$ for 2 of the measurement sequences and $\{I P S, H F M\}$ for 8 of the measurement sequences. Both $\{H F M\}$ and $\{I P S, H F M\}$ are correct diagnoses, but $\{H F M\}$ is more detailed. It would be desirable to always get $\{H F M\}$ as the diagnosis statement. However this is not possible due to the model errors and lack of sufficient excitation in some driving conditions.

In one case, when $\{H F M\}$ is present, an incorrect isolation is obtained, namely $\{M L\}$. The reason for this is again the model errors.

\section{CONCLUSIONS}

In this paper, model based diagnosis of an automotive diesel engine has been considered. The main goal has been to investigate if a model based diagnosis system can be constructed for this engine which is considerably more complex compared to engines in previous studies, e.g. (Nyberg, 1999a; Gertler et al., 1995).

First an accurate model has been constructed. The effects of EGR and VNT are included, and in a validation against a real car, the model was shown to have an error of $3 \%$. However, in the experiments, only one car was used so the individual-to-individual variation could not be studied.

The faults considered were air-mass flow sensor fault, intake-manifold pressure sensor fault, air-leakage between the air-mass flow sensor and the cylinders, and the EGR-valve stuck in closed position. The design of the diagnosis system then follows the framework of structured hypothesis tests. It has been shown that for this application, this framework is a useful engineering tool to systematically design model based diagnosis systems.

In experiments on a real vehicle, driving on the road, the performance of the diagnosis system has been successfully evaluated. Of 66 tested cases, unique isolation was achieved in 52 cases, and non-unique, but correct isolation was achieved in 13 cases. In one case, a fault is detected but incorrectly isolated. To improve the diagnosis system, i.e. to reduce the probability of incorrect isolation, and also non-unique isolation, the model of the engine should be improved. Even though the average error of the model is not so large, it is the worst-case error that causes the problems. It might also be possible to improve the diagnosis system by better tuning, e.g. by using better thresholds.

\section{REFERENCES}

Casella, G. and R.L. Berger (1990). Statistical Inference. Duxbury Press.

Gertler, J., M. Costin, X. Fang, R. Hira, Z. Kowalalczuk, M. Kunwer and R. Monajemy (1995). Model based diagnosis for automotive engines algoritm development and testing on a production vehicle. IEEE Trans. on Control Systems Technology 3(1), 61-69.

Guzzella, L. and A. Amstutz (1998). Control of diesel engines. IEEE Control Systems pp. 53-71.

Heywood, John B. (1992). Internal Combustion Engine Fundamentals. McGraw-Hill series in mechanical engineering. McGraw-Hill.

Höfling, T. and R. Isermann (1996). Fault detection based on adaptive parity equations and single-parameter tracking. Control Eng. Practice 4(10), 1361-1369.

Klingmann., R., W. Fick, H. Brueggemann, D. Naber, K.H. Hoffmann and A. Peters (1999). Die neuen common-rail dieselmotoren mit direkteinspritzung in der modellgepflegten e-klasse. Motortechnische Zeitung, MTZ.

Lehmann, E. L (1986). Testing Statistical Hypotheses. second ed.. Springer Verlag.

Nyberg, M. (1999a). Model based diagnosis of both sensor-faults and leakage in the air-intake system of an SI-engine. SAE Paper 1999-01-0860.

Nyberg, M. (2000). Model based diagnosis using structured hypothesis tests. SAFEPROCESS. IFAC. Budapest, Hungary. pp. 1026-1031.

Nyberg, M. and A. Perkovic (1998). Model based diagnosis of leaks in the air-intake system of an SI-engine. SAE Paper 980514.

Nyberg, Mattias (1999b). Model Based Fault Diagnosis: Methods, Theory, and Automotive Engine Applications. PhD thesis. Linköping University. URL: http://www.fs.isy.liu.se/Publications/.

Truscott, A., A. Noble, A. Cotta and T. Stutte (2000). Simulation of gas path faults in a VGT diesel engine for the development of diagnosis algorithms. SIA International Congress. Lyon, France. pp. 1026-1031. 